\title{
High-Frequency Proximity Losses Determination for Rectangular Cross-Section Conductors
}

\author{
Anh-Tuan Phung, Gérard Meunier, Olivier Chadebec, Xavier Margueron, and Jean-Pierre Keradec \\ Laboratoire d'Electrotechnique de Grenoble, ENSIEG-BP, Grenoble 46-38402, France
}

\begin{abstract}
This paper focuses on proximity losses prediction in conductors of power electronics transformers. The method is based on the concept of equivalent complex permeability applied to rectangular conductors. A 1-D analytical model is analyzed. It has been shown that the 1-D model does not take into account edge effects. Two complementary approaches have been proposed. One treats scattered conductors; other deals with package of conductors. Both approaches are compared with a good accuracy and economic computation time.
\end{abstract}

Index Terms-Finite element analysis (FEA), high frequency (HF), proximity losses, rectangular conductors, transformers.

\section{INTRODUCTION}

I $\mathrm{N}$ power electronics devices, conductors carrying currents are immersed in alternative magnetic field. The current profile is therefore nonuniform, and the effective resistance tends to increase with frequency. The skin effect term is used to illustrate nonuniformity in a single conductor, caused by its own magnetic field. The proximity effect is applied for nonuniformity caused by magnetic fields of adjacent conductors. Studies on eddy current effects show that proximity effect is predominant in high frequency (HF) [1], [2]. Therefore, correct prediction on proximity effect losses is required for an accurate magnetic component design.

Analytical solution [3] for eddy current problems is limited to simple geometry-like circular cross-section conductors or infinite wide strip. Hence, numerical analysis is intensively used for arbitrary geometry. For finite element analysis (FEA), one among criterions required to obtain a sufficient reliable result is to have at least two elements in skin depth. When dealing with 3-D devices modeling like transformers or inductors simulation, this constraint is not easily satisfied. In most cases, it reveals unfeasible to carry out a complete 3-D simulation with standard mesh density. The memory requirement and computation time become rapidly prohibitive.

Many studies show that it is interesting to use complex permeability to predict proximity losses. It has been successfully applied to calculate proximity losses in transformers with circular cross-section conductors [4]. Complex permeability computation for rectangular conductors with analytical formulas [5] uses 1-D assumption. Hence, its application domain is restricted to conductors with large aspect ratio (length per width) and homogenous tangential field.

In this paper, we propose to determine 2-D complex permeability numerically. Section II reviews principle of complex permeability and related hypothesis. 1-D model with closedform formulas is also exposed. Section III will focus on 2-D

Digital Object Identifier 10.1109/TMAG.2007.892303

Color versions of one or more of the figures in this paper are available online at http://ieeexplore.ieee.org.

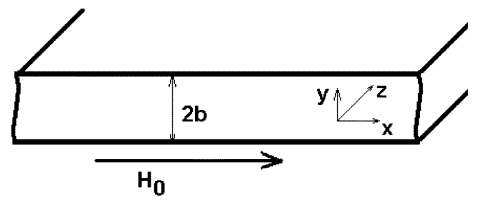

Fig. 1. The 1-D model configuration. External magnetic field $H_{0}$ is not supposed to be disturbed by the presence of the conducting plate of thickness $2 b$.

edge effect and numerical complex permeability determination. Section IV finalizes with 2-D and 3-D validation.

\section{Complex Permeability-1-D Analytical Model}

\section{A. Principle}

To compute proximity losses without involving a huge mesh, complex permeability concept is employed. The principle consists of replacing the conductor material by an equivalent nonconductive magnetic material, which gives the same active and reactive power loss for a given geometry. There is no more eddy currents loss computation which is time consuming. The intense mesh is replaced by a coarse one. In fact, the number of unknowns in conducting regions is divided by four because only one unknown per node is required (basically, four unknowns are required). Frequency-dependent ohmic losses are included by a phase shift between magnetic field and magnetic induction.

First, we look at the 1-D model and its assumptions.

\section{B. 1-D Complex Permeability Model}

Let us consider a conducting infinite plate of $2 b$ thickness in a homogenous tangential field $H_{0}$ in Fig. 1.

The external field is considered to be not disturbed by the presence of the plate. Writing the field expression in the plate, the only component is

$$
H(y)=H_{0} * \frac{\cosh (\alpha y)}{\cosh (\alpha b)} \text { with } \alpha=\frac{1+j}{\delta} \quad \text { and } \delta=\sqrt{\frac{2}{\omega \mu \sigma}} .
$$

The instantaneous complex power density is written as

$$
\begin{aligned}
p & =\frac{1}{\sigma} \mathbf{J} \overline{\mathbf{J}}+j \mu \omega \mathbf{H} \overline{\mathbf{H}} \\
& =\mu \omega H_{0}^{2}\left(\frac{\cosh (Y)-\cos (Y)}{\cosh (B)+\cos (B)}+j \frac{\cosh (Y)+\cos (Y)}{\cosh (B)+\cos (B)}\right)
\end{aligned}
$$




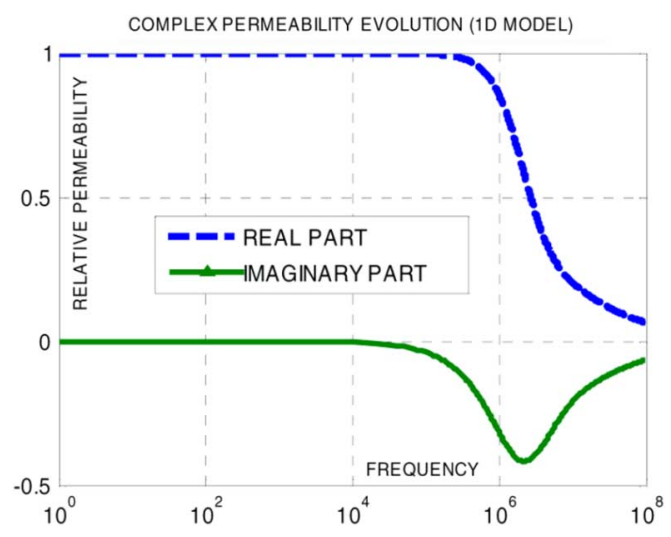

Fig. 2. Complex permeability evolution. External field along the conducting plate. Plotting of the $\mu_{Y}^{*}$ with $2 b=0.1 \mathrm{~mm}, \sigma=0.058 \mathrm{~S} / \mathrm{cm}$ (copper).

with

$$
Y=\frac{2 y}{\delta} \quad \text { and } \quad B=\frac{2 b}{\delta}
$$

which leads to the complex power by unit length

$$
\begin{aligned}
P= & \int_{-b}^{+b} p d y=\sqrt{\frac{2 \mu \omega}{\sigma}} H_{0}^{2} \\
& \times\left(\frac{\sinh (B)-\sin (B)}{\cosh (B)+\cos (B)}+j \frac{\sinh (B)+\sin (B)}{\cosh (B)+\cos (B)}\right) .
\end{aligned}
$$

By replacing this conducting plate with a nonconductive magnetic plate, the instantaneous complex power density will be characterized by a hysteresis cycle $B=\mu^{*} H$. The magnetic field inside the plate is uniform and takes value of $H_{0}$.

The complex power density will have an active and a reactive component which is written as

$$
p=j \mu^{*} \omega \mathbf{H} \overline{\mathbf{H}}=\left(\Re\left(\mu^{*}\right)+j . \Im\left(\mu^{*}\right)\right) j \omega H_{0}^{2} .
$$

Integrating (4) over the width of the plate and equaling this with (3), we obtain finally the expression of the complex permeability. This equivalent permeability depends on working frequency and nature of the plate (dimension, conductivity, permeability, etc.)

$$
\begin{aligned}
& \Re\left(\mu^{*}\right)=\frac{\mu \delta}{2 b}\left(\frac{\sinh (B)+\sin (B)}{\cosh (B)+\cos (B)}\right) \\
& \Im\left(\mu^{*}\right)=-\frac{\mu \delta}{2 b}\left(\frac{\sinh (B)-\sin (B)}{\cosh (B)+\cos (B)}\right) .
\end{aligned}
$$

The complex permeability evolution as a function of frequency is presented in Fig. 2.

The expression (5) is quite easy to implement into a proximity loss calculation program. However, its application is limited in 1-D. In [5], authors have intended to use this model for 2-D simulations by rotating the device an angle of $90^{\circ}$ and applying the same boundary condition. In other words, authors have used two 1-D models to derive the anisotropy of complex permeability $\left(\mu_{X}^{*}\right.$ and $\left.\mu_{Y}^{*}\right)$. This leads to significant relative errors at some sufficient high frequencies.

In fact, the existence of a second component of the magnetic field (which is orthogonal to the conducting plate) modifies the

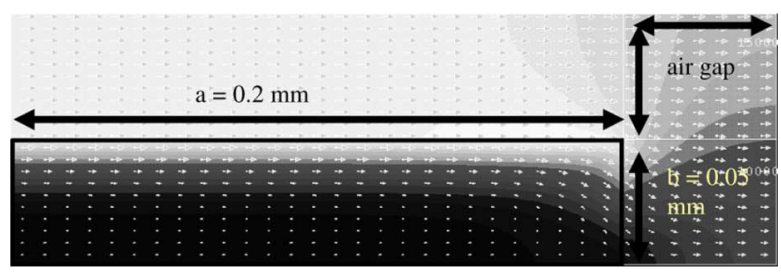

Fig. 3. Long conductive plate under tangential field. Apparition of orthogonal component magnetic field. Located edge effect. Dimension $0.4 \times 0.1 \mathrm{~mm}$; air-gap $=0.1 \mathrm{~mm}$. A quarter of geometry is described.

HF ohmic losses. Moreover, with a limited length in 2-D model, current density is typically located at the edges of the device [6]. These two factors could not to be taken into account by a 1-D model (see Fig. 3).

To our knowledge, there is not analytical treatment yet for 2-D foils. To include the edge effects into complex permeability calculation, we propose two numerical approaches. The first deals with isolated conductor and is coupled with homogenization technique. The second treats the case of the large number of conductors arranged in a regular manner.

\section{NumericAl COMPLEX PERMEABILITy DeterminAtion}

\section{A. Isolated or Scattered Conductors}

First, the isolated rectangular conductor is placed in a uniform alternative inductor field. 2-D finite element (FE) eddy currents problem is solved and active and reactive powers are computed ( $P_{\text {ref }}$ and $Q_{\text {ref }}$ ). This stage could be time consuming because we must solve eddy current problem for each frequency. However, it stays reachable.

The external field must be applied at both tangential and perpendicular directions. These two directions are necessary because of the anisotropy of the complex permeability. At the end of this stage, we obtain four values of active and reactive power $\left(P_{\text {ref }}^{X}, Q_{\text {ref }}^{X}\right.$ and $P_{\text {ref }}^{Y}, Q_{\text {ref }}^{Y}$, respectively).

Second, the conductive material will be replaced by a magnetic material with complex permeability. An optimization solver [global optimization toolbox (GOT)] [7] will drive the simulation with complex permeability-like input data in order to match the active and reactive power reference. The multiobjective function for direction $X$ of field is

$$
f_{P}^{X}=\frac{\left(P-P_{\mathrm{ref}}^{X}\right)^{2}}{\left(P_{\mathrm{ref}}^{X}\right)^{2}} \quad \text { and } f_{Q}^{X}=\frac{\left(Q-Q_{\mathrm{ref}}^{X}\right)^{2}}{\left(Q_{\mathrm{ref}}^{X}\right)^{2}} .
$$

We have a similar expression for other direction of field.

The problem does not need an intense mesh because there is no more eddy current in the complex magnetic material. However, it could be time consuming, depending on how fine the variation interval is divided. In effect, in order to fit $P_{\text {ref }}$ and $Q_{\text {ref }}$, GOT will vary the permeability over its variation space (which is usually normalized to $[0,1]$ interval) to construct a response surface (RS). The quality of RS depends on the number of points of computation. The more number of points of computation, the better the response surface is. For case presented in Table I, we have used ten points of division for each interval. Hence, the total computation is $2 \times 10 \times 10=200$ simulations.

This response surface will be exploited by GOT using genetic algorithm to derive a Pareto front from which we select an op- 
TABLE I

COMPARISON OF 1-D ANALYTICAL MODEL With DiRECT IDENTIFICATION

\begin{tabular}{|c|c|c|}
\hline & \multicolumn{2}{|c|}{$\begin{array}{l}\text { VALUE OF COMPLEX } \\
\text { PERMEABILITY }\end{array}$} \\
\hline & Real part & Imaginary part \\
\hline Direct identification by GOT & 0,999992986 & $-0,007829731$ \\
\hline \multirow[t]{3}{*}{ I-D analytical model } & 0,999853715 & $-0,00764802$ \\
\hline & \multicolumn{2}{|c|}{ VALIDATION ON 2D EXAMPLE } \\
\hline & $\begin{array}{l}\text { Relative error on } \\
\text { active power } \mathrm{P}\end{array}$ & $\begin{array}{l}\text { Relative error on } \\
\text { reactive power Q }\end{array}$ \\
\hline Direct identification by GOT & $0.2 \%$ & $0.03 \%$ \\
\hline 1-D analytical model & $3.8 \%$ & $0.04 \%$ \\
\hline
\end{tabular}

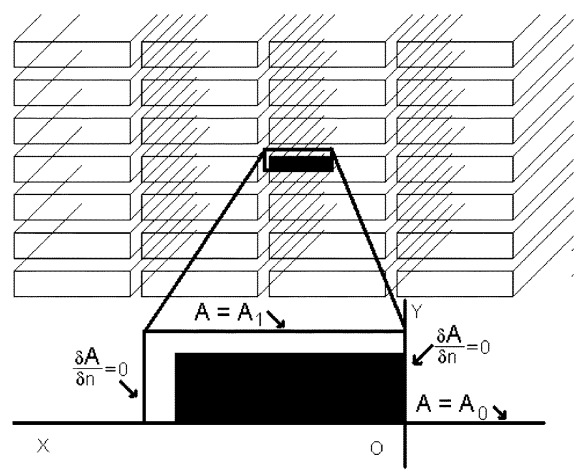

Fig. 4. Model for package of conductors. The elementary cell with appropriate boundary conditions.

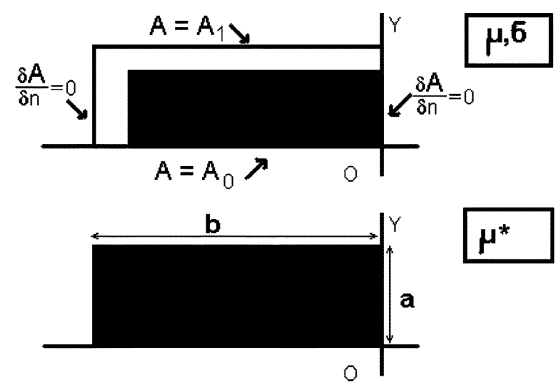

Fig. 5. Elementary cell model with imposed boundary conditions.

timum. This optimum corresponds to the combination of complex permeability which matches the best $P_{\text {ref }}$ and $Q_{\text {ref. }}$. This latter combination is now ready for use in 2-D FE simulation with an inexpensive computation cost.

In this approach, we can obtain a combination of complex permeability which could return exact proximity losses for each frequency for a scattered conductor. However, it is not straightforward while we deal with multiple frequencies. Second, when applying to case of bundle of conductors, the basic reluctance network does not take into account interaction between conductors. The homogenized complex permeability is therefore far from its ideal condition. To handle this obstacle, we propose to deal with package of conductors (see Fig. 4).

\section{B. Package of Conductors Elementary Cell Model}

Our hypothesis is that there are an infinite number of straight rectangular conductors immersed into a homogenous external field. In this case, it is clear that the reaction field of each small

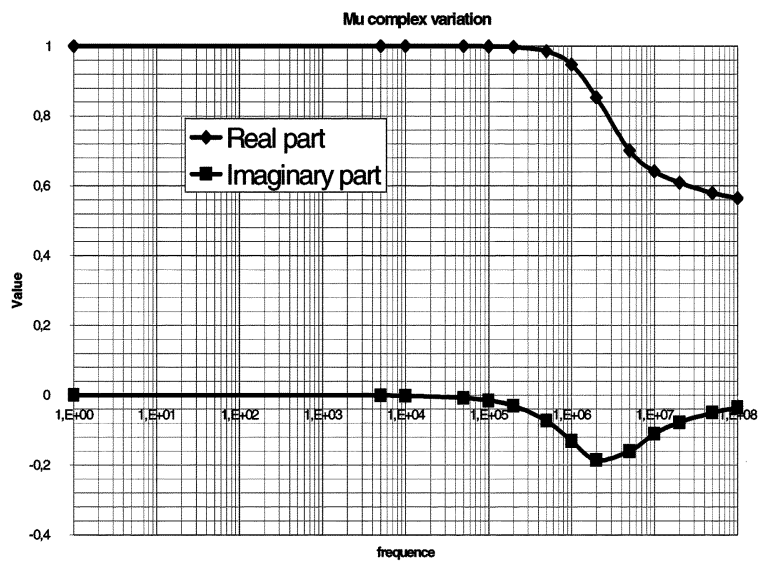

Fig. 6. Model for package of conductors. External field along the conducting plate. Plotting of the $m u_{Y}^{*}$ with $2 b=0.1 \mathrm{~mm} . \sigma=0.058 \mathrm{~S} / \mathrm{cm}$ (copper).

conductor does not modify the external field. The external field could be vertical or horizontal. We consider only one quarter of the elementary cell because of the symmetry.

In Fig. 5, we describe how to create a horizontal field along the conductor. Vector potentials are imposed on top and bottom; Neumann conditions are imposed to force the field rigorously tangential to Ox. By their nature, these boundary conditions also create a periodicity repeated infinitely in two directions. On this greatly reduced size of the cell, we computed the active and reactive power for each frequency

$$
W(f)=P(f)+j Q(f) .
$$

These values will be then equalized to those we obtain from the complex permeability model. Let us now look at the equivalent complex permeability model. The magnetic induction and magnetic field are calculated by

$$
B_{0}=\frac{\Delta A}{a} \quad H_{0}=\left(\nu^{\prime}+j \nu^{\prime \prime}\right) B_{0} .
$$

The instantaneous complex power is integrated and equalized to (7)

$$
p=j\left(\nu^{\prime}+j \nu^{\prime \prime}\right) \omega B_{0}^{2} .
$$

Hence, we can obtain the expression of the complex permeability versus frequency

$$
\mu^{\prime}+j \mu^{\prime \prime}=\frac{1}{\nu^{\prime}+j \nu^{\prime \prime}}
$$

with

$$
\nu^{\prime}=\frac{1}{\omega B_{0}^{2} a b} Q(f) \quad \nu^{\prime \prime}=\frac{-1}{\omega B_{0}^{2} a b} P(f) .
$$

The evolution of complex permeability versus frequency is given in Fig. 6. This method is quite simple to execute. The result is obtained with precision and over a large band of frequencies. We are going to validate both methods on 2-D and 3-D examples.

\section{VALIDATION}

\section{A. 2-D Validation}

Both proposed methods have been tested on a simple 2-D example. We evaluate proximity losses caused by inductor over 


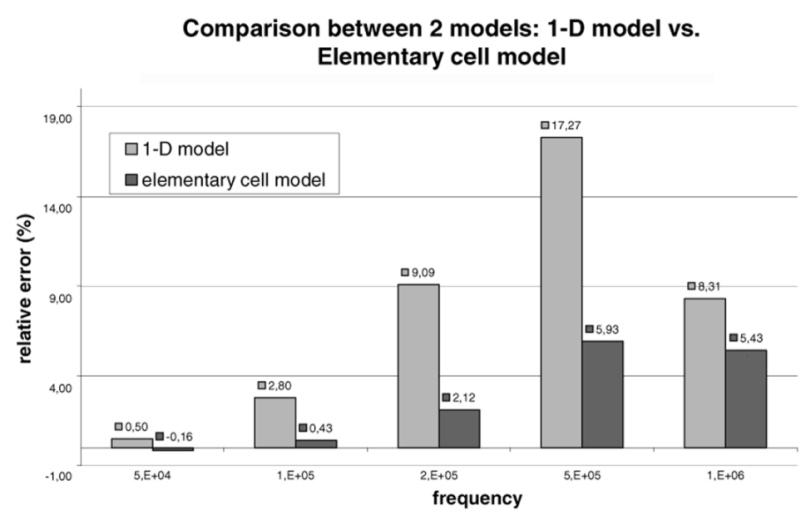

Fig. 7. Relative error comparison on power losses calculation between two models. The same device dimension as in Table I.
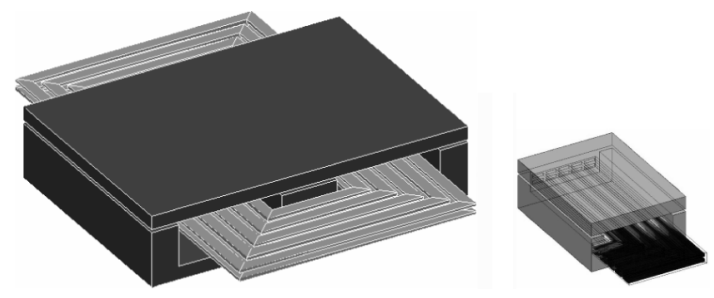

Fig. 8. Geometry of the transformer test. One quarter is described. Secondary conductors are in open circuit. Primary and secondary conductors are situated in parallel.

TABLE II

COMPARISON ON A 3-D EXAMPLE AT $500 \mathrm{kHz}$

\begin{tabular}{llll}
\hline \hline & POWER LOSS & $\begin{array}{l}\text { COMPUTATION } \\
\text { TIME }\end{array}$ \\
\hline & Active & Reactive & \\
\hline $\begin{array}{l}\text { Direct computation - } \\
\text { Eddy current problem }\end{array}$ & $1.505 \mathrm{~mW}$ & $7.07 \mathrm{mVAr}$ & 12 hours \\
\hline $\begin{array}{l}\text { Equivalent complex } \\
\text { permeability model }\end{array}$ & $1.664 \mathrm{~mW}$ & $7.11 \mathrm{mVAr}$ & $\begin{array}{l}1 \text { minute } 30 \\
\text { seconds }\end{array}$ \\
\hline
\end{tabular}

bundle of conductors in a transformer. Conductors dimension are $0.4 \times 0.1 \mathrm{~mm}$ with $0.1-\mathrm{mm}$ air-gap between them. Working frequency varies from $50 \mathrm{kHz}$ to $1 \mathrm{MHz}$. All results are compared to standard eddy currents problem. For direct identification model, despite of its high precision, this model demands relatively large number of simulations. Therefore, graphical presentation of relative error versus frequency of this model is not preferred. We present only comparison between the 1-D analytical model and the elementary cell model (see Fig. 7). We can state that a strong improvement in term of precision has been realized.

\section{B. 3-D Validation}

These methods have been applied for 3-D simulations. In fact, the third component of a magnetic field does not have much influence in most configurations. Primary and secondary conductors are generally situated in parallel. For this reason, 2-D complex permeability remains applicable for 3-D proximity losses determination.
A 3-D transformer test is presented in Fig. 8. Its secondary circuit has only two layers of five conductors. Because of the symmetry, we describe only one quarter of its geometry. Working frequency varies from 50 to $500 \mathrm{kHz}$. A short resume is presented in Table II. The transformer is modeled using a commercial simulation code [8]. Secondary conductors stay in open-circuit status to study proximity losses caused by its primary coils. To properly take into account proximity losses in conductor, the eddy current problem is modeled with 730000 elements. Total computation time for each frequency is about $12 \mathrm{~h}$ on a Pentium IV $2.8 \mathrm{GHz}, 2 \mathrm{~GB}$ of live memory. With predetermined values of 2-D complex permeability, an equivalent complex permeability problem is modeled. The equivalent problem takes 350000 elements. Computation time is drastically reduced to $90 \mathrm{~s}$. The relative error is under $5 \%$ from 50 to $250 \mathrm{kHz}$. The worst case is at $500 \mathrm{kHz}$ where relative errors are about $9.5 \%$ for active power calculation. But in this case, the precision limits of the eddy current problem are reached and are due to the limits of computation capabilities.

\section{CONCLUSION}

We have presented two complementary numerical approaches to predict proximity losses in rectangular conductors. The first method treats the case of isolated conductors. It is useful when modeling conductors which are far from others. The second deals with package of conductors using elementary cell. This method is truly practical when conductors are arranged similarly. Both two methods have been tested on simple 2-D and 3-D examples. It has been proved that good accuracy and reduced computation time are acquired. These two methods facilitate greatly proximity losses prediction for HF design.

\section{ACKNOWLEDGMENT}

The authors would like to thank Prof. J.-L. Coulomb from Laboratoire d'Electrotechnique de Grenoble for his help with the optimization tool GOT.

\section{REFERENCES}

[1] A. W. Lofti and F. C. Lee, "Proximity losses in short coils of circular cylindrical windings," in Proc. IEEE Power Electron. Specialists Conf., Jul. 1992, vol. 2, pp. 1253-1260.

[2] M. Albach, "Two-dimensional calculation of winding losses in transformers," in Proc. IEEE Power Electron. Specialists Conf., Jun. 2000, vol. 3, pp. 1639-1644.

[3] J. Lameraner and M. Stafl, Eddy Currents. London, U.K.: Illife Books, 1966

[4] E. Matagne, "Modélisation magnétique macroscopique des faisceaux de conducteurs," in J. Phys. III, France, 1993, vol. 3, pp. 509-517.

[5] O. Moreau, L. Popiel, and J. L. Pages, "Proximity losses computation with a 2D complex permeability modelling," IEEE Trans. Magn., vol. 34, no. 5, pp. 3616-3619, Sep. 1998.

[6] N. Dai and F. C. Lee, "Edge effect analysis in a high-frequency transformer," Virginia Power Electronics Center, Blacksburg, VA, 1994.

[7] J. L. Coulomb, "Electromagnétisme et problémes couplés," ElectromagnéTisme et éLéments Finis 3, 2002.

[8] $F L U X^{\circledR}$, Software solutions [Online]. Available: http://www.cedrat.com/software/flux

Manuscript received October 27, 2006 (e-mail: Gerard.Meunier@leg.ensieg. inpg.fr). 